\title{
Products and Processes of Ionization in Methyl Chloride as Determined by a Mass Spectrometer
}

\author{
S. H. BAueR* AND T. R. Hogness, George Herbert Jones Laboratory, University of Chicago
}

(Received August 30, 1935)

\begin{abstract}
The following ions were found to be produced in methyl chloride as primary products: $\mathrm{CH}_{3} \mathrm{Cl}^{+}, \mathrm{CH}_{3}+, \mathrm{CCl}^{+}, \mathrm{CH}_{2}+, \mathrm{Cl}^{+}, \mathrm{HCl}^{+}, \mathrm{CH}^{+}$and $\mathrm{C}^{+}$- listed in order of decreasing intensity. Small concentrations of the $\mathrm{H}^{+}$ion were also detected. This latter peak was not investigated completely. The appearance potentials for the $\mathrm{CH}_{3} \mathrm{Cl}^{+}, \mathrm{CH}_{3}{ }^{+}$and $\mathrm{Cl}^{+}$ions are 11.0, 14.7 and 26 volts, respectively.
\end{abstract}

I.

$\mathbf{M}^{\mathrm{A}}$ ASS spectrographic studies of organic compounds in which the ions were generated by electrons of known energy, have not been very extensive. From among the gases thus far studied, ${ }^{2}$ the appearance potentials for the ions formed in acetylene ${ }^{2}$ and methane ${ }^{3}$ only have been determined. Since under most circumstances of ionization many different kinds of fragments appear as a result of primary processes, it is evident that unless these potentials are known, the mechanisms of dissociation and ionization must remain uninterpreted. The work reported in this paper was initiated with the hope that the results obtained would be of some value in giving an estimate for the heat of reaction of

$$
\mathrm{CH}_{3} \mathrm{Cl}=\mathrm{CH}_{3}+\mathrm{Cl} \text {. }
$$

Such an estimate could only be obtained from a knowledge of the energy necessary for the reaction

$$
\mathrm{CH}_{3} \mathrm{Cl}=\mathrm{CH}_{3}+\mathrm{Cl}^{+}+e^{-} \text {. }
$$

This process does not occur to any appreciable extent when methyl chloride is bombarded by electrons.

* Present address: Gates Chemical Laboratory, California Institute of Technology.

1 R. Conrad and co-workers, Trans. Faraday Soc. 30 215 (1934); E. G. Linder and A. P. Davis, J. Phys. Chem. 35, 3649 (1931). The above authors used an electric discharge for their ion source. In the following work controlled electron excitation was employed: H. R. Stewart and A. R. Olson, J. Am. Chem. Soc. 53, 1236 (1931), studied propane and butane; E. G. Linder, Phys. Rev. 41, 149 (1932), benzene: J. Chem. Phys, 1,129 (1933), octane.

${ }^{2}$ J. T. Tate, P. T. Smith and A. L. Vaughan, Phys. Rev. 43, 1054A (1933); Phys. Rev. 48, 525 (1935).

3 T. R. Hogness and H. M. Kvalnes, Phys. Rev, 32, 942 (1928); J. H. Hipple and W. Bleakney, Phys. Rev, 47, $802 \mathrm{~A}(1935)$.
II.

The apparatus constructed was of the Dempster type as modified by Hogness and Lunn and somewhat further in the present investigation. ${ }^{4}$ A section is shown in Fig. 1. A flow system, regulated by means of a low pressure variable gas leak, was set up through the ionization chamber while the pressures in the other compartments were maintained at a low value by means of high speed mercury diffusion pumps. ${ }^{5}$ The following details may be of interest.

(1) The radius of curvature of the path of the ions in the magnetic field was $6.35 \mathrm{~cm}$. The collimating slits $S_{2}$ and $S_{3}$ as well as the receiving slit in front of the collecting plate (not shown) were all $0.025 \mathrm{~cm}$. Further, the pressures in the chambers 3 and 4 were very low (about $10^{-5}$ and $10^{-6}$, respectively) and the refocusing fairly good. Hence, the resolution was such as to permit easily the detection of differences of mass of the order $\Delta m / m=0.006$. This can be ascertained from Fig. 2 which is a plot of one of the earlier runs. ${ }^{6}$

(2) A Compton electrometer (sensitivity 40,000 to 2000 deffections per volt), whose insulated quadrants were grounded through an "iodine in benzene" resistance ${ }^{7}$ of $4 \times 10^{11} \mathrm{ohms}$, was used as the current measuring device.

(3) The slit $S_{1}$ had the dimensions $0.2 \times 0.6$ $\mathrm{cm}$. This size was found necessary in order that a

4 T. R. Hogness and E. G. Lunn, Phys. Rev. 26, 44 (1925) and subsequent work. The following authors used analyzers modified along lines similar to the present apparatus: H. Kallmann and B. Rosen, Zeits. f. Physik 64, 806 (1930); O. Luhr, Phys. Rev, 44, 459 (1933).

$s$ Following the design of T.E. Phipps, private communication.

${ }^{6}$ The peaks at $40: 32: 29.4,28: 18$ and 16 are due, respectively, to $\mathrm{A}^{+}: \mathrm{O}_{2}{ }^{+}: \mathrm{N}_{2}{ }^{+}: \mathrm{H}_{2} \mathrm{O}^{+}$and $\mathrm{O}^{+}$which were present in the apparatus before the methyl chloride was put in.

${ }_{7}$ Rollefson, Science 60, 226 (1924). 


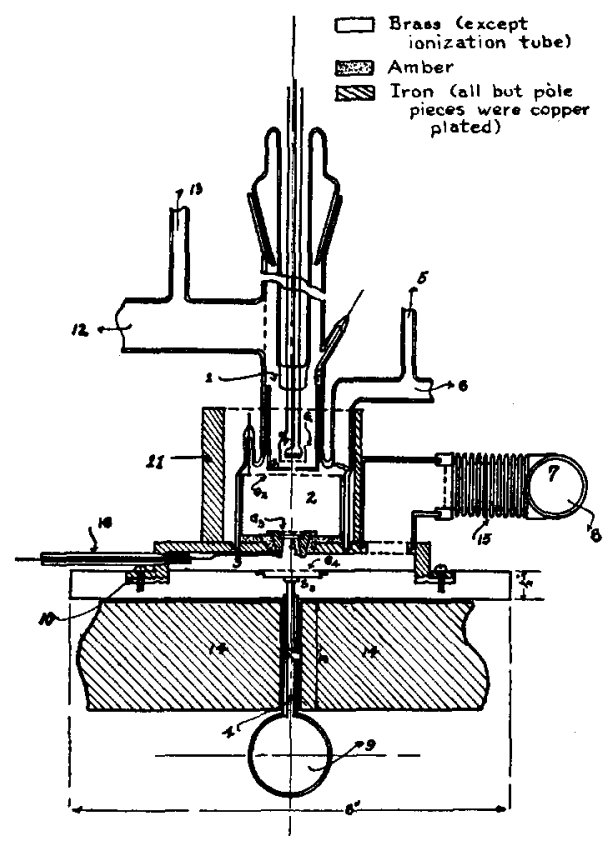

FIG. 1. Side view of the apparatus.

(1) Filament chamber

(2) Ionization chamber

(4) Deflection chamber

(5) To leak No 1

(6). (8). (9), (13) To valves and

. (8)Leod gauge

(7) Metal to glass ground joint (8) To leak No. 2 ; also to pump
No. 2

\author{
(9) To charcoal trap and pump \\ No. 3 \\ (10) Rubber gasket \\ (ii) Laminated shielding \\ (12) To pump shicid \\ (14) Pole pieces \\ (15) Bendix \\ (16) Pyrex
}

sufficient number of electrons would be permitted to enter compartment 2. Nevertheless, because of an efficient pumping system, the pressure in the filament chamber (1) was maintained at $1 / 20$ of that in the ionization chamber. Since by experiment it was determined that the identical peaks were obtained with and without such a differential pumping arrangement in spite of the fact that dissociation on the surface of the filament was found in the latter case, this differential pressure was deemed sufficient.

(4) The performance of the apparatus was satisfactory except for one feature-the presence of ghost peaks at 0.04 to 0.05 mass unit above the primary ones, as shown by $54,29.4$ and 15.7 in Fig. 2 and by 2.1 in Fig. 3. This was very troublesome at first, when both peaks appeared to have about the same intensity. After a series of modifications in the slit system the magnitude of the ghost was reduced to a small value (but could not be eliminated entirely) so that only the large peaks exhibit this structure. The doubling may have been caused either by the peculiar electric field around $S_{2}$ or by reflection of the ion beam on some part of the walls of the deflection chamber (4). Since the ghost peaks did not interfere with the experiments while the elimination of both these possibilities would have necessitated the reconstruction of the entire apparatus, no further alterations were made at this time.

\section{III.}

From Figs. 2 and 3 it is evident that all the possible positive ions which may result from the dissociation of methyl chloride, except $\mathrm{CH}_{2} \mathrm{Cl}^{+}$ and $\mathrm{CHCl}^{+}$, appeared. These may have been present in such small quantities as to be entirely obscured by the large peaks at 52 and 50 . The $\mathrm{H}^{+}$peak due to mo iyl chloride (Fig. 3) was obtained with $45 \mathrm{v}$ electrons at a pressure of $2 \times 10^{-3} \mathrm{~mm}$ of $\mathrm{Hg}$ in the ionization chamber. Because of its small intensity and the difficulty of working in this mass range when all the rest of the ions were in an entirely different portion of the scale, this peak was investigated no further. There is no doubt that its appearance potential is quite high (perhaps around $30 \mathrm{v}$ ). ${ }^{8}$ All the other ions were generated as primary products, as is indicated by the curves in Fig. 4. Their relative intensities depended to some extent on the pressure; roughly in the manner given in Table I. Since none of the curves in Fig. 4 extrapolate to zero intensity for zero pressure, these ions are the results of primary dissociations by electron impact. Multiply charged ions were not observed and would hardly have been expected at low voltages used $(33.0-45.0)$ for the bombarding electrons.

TABLE I.

\begin{tabular}{lc|lc}
\hline \hline IoN & $(0.08-6.5) \times 10^{-3} \mathrm{~mm}$ & \multicolumn{1}{c}{ loN } & $(0.08-6.5) \times 10^{-3} \mathrm{~mm}$ \\
\cline { 2 - 4 } $\mathrm{CH}_{3} \mathrm{Cl}^{+}$ & $56.0-31.0$ & $\mathrm{Cl}^{+}$ & $4.0-8.0$ \\
$\mathrm{CH}_{3}{ }^{+}$ & $24.0-45.0$ & $\mathrm{HCl}^{+}$ & $3.0-3.0$ \\
$\mathrm{CCl}^{+}$ & $5.5-6.0$ & $\mathrm{CH}^{+}$ & $2.2-2.0$ \\
$\mathrm{CH}^{+}$ & $4.2-4.3$ & $\mathrm{C}^{+}$ & $1.0-0.6$ \\
\hline
\end{tabular}

${ }^{8}$ In methane, Hipple and Bleakney (reference 3 ) give for the appearance potentials of $\mathrm{CH}_{4}^{+}, \mathrm{CH}_{3}{ }^{+}, \mathrm{CH}_{2}{ }^{+}, \mathrm{CH}^{+}$, $\mathrm{C}^{+}$, and $\mathrm{H}^{+}$the values $13.7,14.7,15.7,23,27$ and 31 , respectively. In methyl chloride one would expect quite similar potentials for the last four ions. 


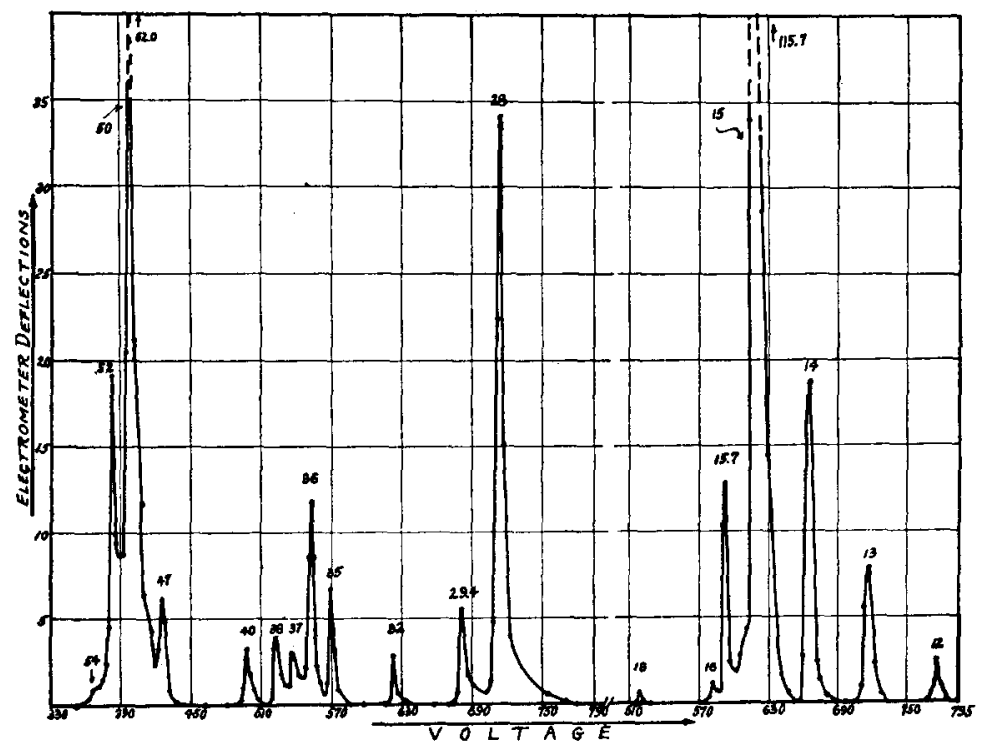

FIG. 2. Mass spectrum of methyl chloride plus residual air.

The appearance potentials for three of the ions were determined. They are: $\mathrm{CH}_{3} \mathrm{Cl}^{+} 11.0 \pm 0.5$; $\mathrm{CH}_{3}+14.7 \pm 0.3 ; \mathrm{Cl}+26.5 \pm 1$. The value obtained for the ionization potential of methyl chloride may be compared with the one given by $\mathrm{T}$. N. Jewitt $^{9}(10.7 \mathrm{v})$ who used molecular ray technique with a Kingdon cage arrangement for the determination, and with the spectroscopic values of W. C. Price ${ }^{10}(11.17$ and $11.25 \pm 0.01$ v) who found for methyl chloride two electronic series of absorption bands in the far ultraviolet, going respectively to the above limits. The series conformed closely to a Rydberg formula.

Particular efforts were extended to obtain an accurate estimate of the appearance potential of the $\mathrm{CH}_{3}{ }^{+}$. The value quoted above is the mean of seven determinations carried out under various conditions. The critical voltages for the others were not considered significant enough to warrant the trouble of the determination since the processes of their formation is complicated and the fragments come off with considerable excitational energy. Thus, one can predict these potentials to be relatively high. ${ }^{11}$

${ }^{3}$ T. N. Jewitt, Phys. Rev. 46, 616 (1934).

${ }_{10}$ W. C. Price, Phys. Rev. 47, 510L (1935).

11 See Note 8 . In general, for a single substance, a rough idea of the relative magnitude of the appearance potentials

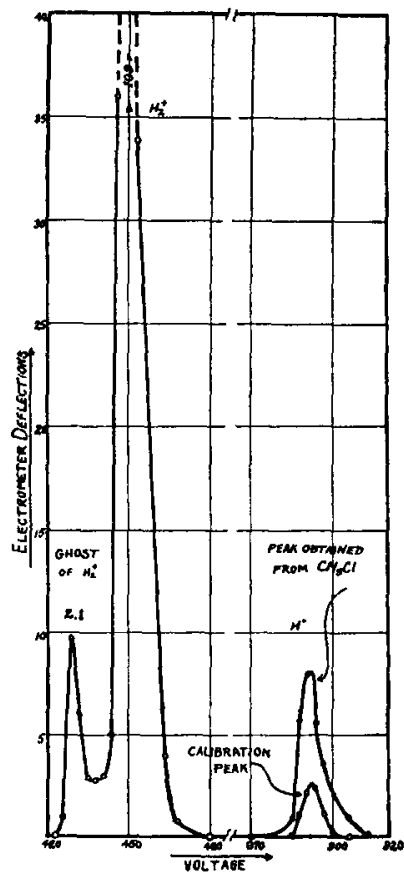

FIG. 3. The $\mathrm{H}^{+}$peak of methyl chloride. Hydrogen is used as a calibrating gas. 


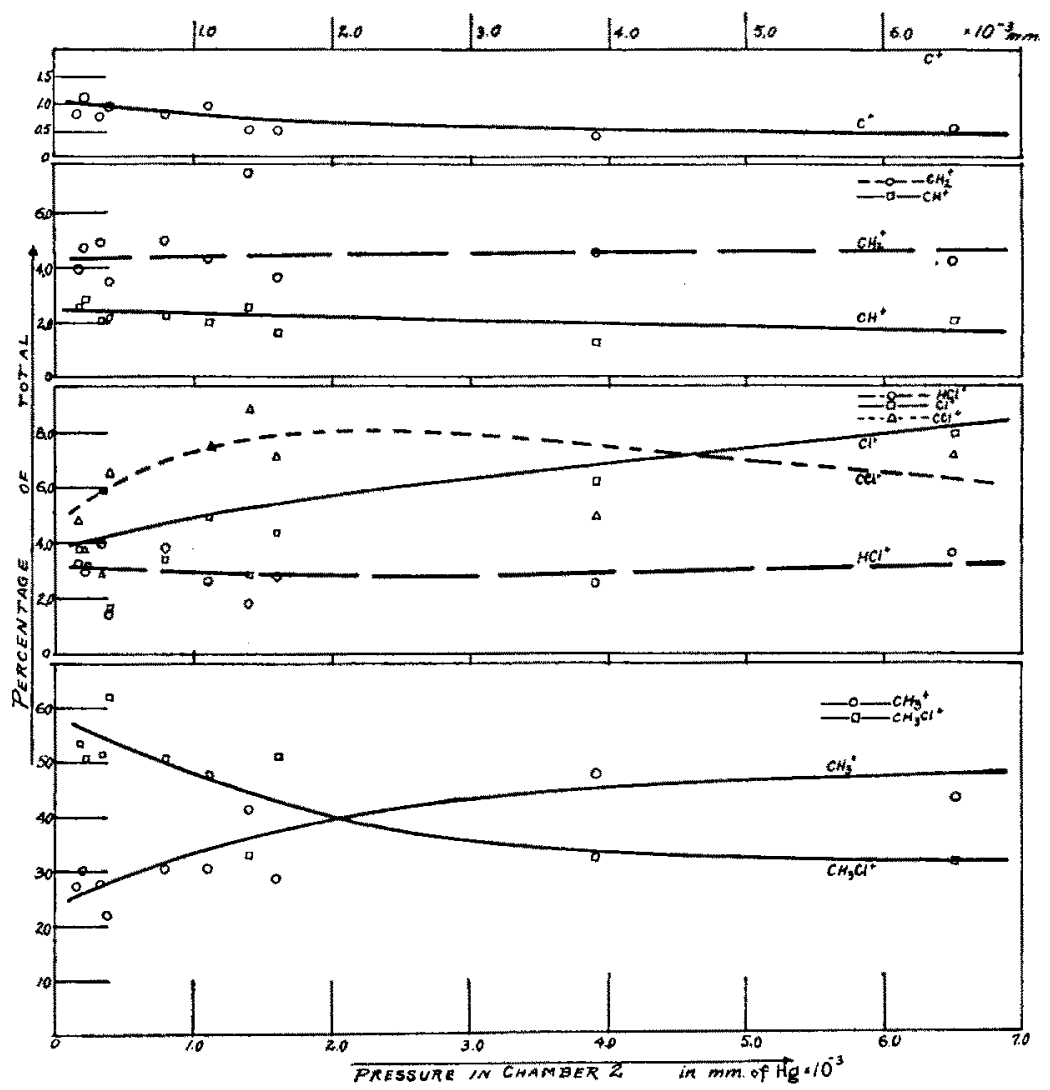

FIG. 4. Variation of the relative intensities of the ion peaks with the pressure. Note change in scale in the three upper sections of the plot.

\section{Details of the experimental procedure}

(1) The methyl chloride used was condensed from a tank (Ohio Chemical Company specifications give it as 99.5 percent pure) by liquid air. The sample was then kept at its melting point while pumping from it continued until its volume was reduced to about $3 / 4$ of its original value thus insuring removal of any contaminating hydrogen chloride and other gases of low boiling point. The remainder was then fractionally distilled in vacuum. Only the middle fraction was used.

(2) In the determinations of the variation of intensity with the pressure the necessary integra-

for the different ions can be obtained from the relative intensities of the peaks when the gas is bombarded with electrons whose velocities are well above the highest of the potentials. tions were accomplished by tracing all the curves plotted on the same scale on a uniform heavy grade of drawing paper, cutting out the individual peaks and weighing them. A large error is inherent in any form of integration of individual narrow, overlapping peaks, particularly when they are unsymmetrical so that their resolution is very uncertain. Hence the data check as well as can be expected. When these runs were made the electron emission and voltage were kept constant at $4.0 \mathrm{ma}$ and $45.0 \mathrm{~V}$, respectively.

(3) Before or after each appearance potential determination, a calibration of the apparatus was made using argon as the calibrating gas. The relative pressures of the two gases were adjusted so that under the identical conditions the argon 
peak and the one being investigated had the same height $( \pm 5$ percent) when the incident electrons were accelerated to 45 volts. This potential was then decreased and the extrapolation made in the usual manner.

IV.

The ionization reaction requiring the minimum energy may be written

$$
\mathrm{CH}_{3} \mathrm{Cl}=\mathrm{CH}_{3} \mathrm{Cl}^{+}+e^{-}+11.2 \mathrm{ev}
$$

and interpreted, according to R. S. Mulliken, ${ }^{12}$ as involving the removal of one of four $3 p \pi_{\mathrm{cl}}$ electrons (nonbonding), leaving the molecule in a ${ }^{2} E$ state. This explains the presence of two Rydberg series limits in the work of W. C. Price ${ }^{10}$ corresponding to the doublet of levels of the final configuration. Obviously the mass spectrograph is too crude an instrument to distinguish the difference between the two.

As was intimated in the introduction, it had been hoped that the products of the reaction

$$
\mathrm{CH}_{3} \mathrm{Cl}=\mathrm{CH}_{3}+\mathrm{Cl}^{+}+e^{-}
$$

should be produced without involving considerable excitational energy. If that were so, the bonding energy of $\mathrm{C}-\mathrm{Cl}$ could be determined since the ionization potential of $\mathrm{Cl}$ is known $(12.96 \mathrm{v})$. This would require the appearance potential of $\mathrm{Cl}$ to be in the vicinity of 18 volts. ${ }^{13}$

12 R. S. Mulliken, Phys. Rev. 47, 413 (1935).

${ }_{13}$ The available data on the heats of combustion of methane and methyl chloride gave the values for the bonding energies of $\mathrm{C}-\mathrm{H}$ and $\mathrm{C}-\mathrm{Cl}$ as 4.11 and 3.14 e.v., respectively. The sources are: Landolt-Börnstein Tables, fifth edition, original volume. W. Jevons, Report on BandSpectra of Diatomic Molecules (Cambridge, 1932); G. B. Kistiakowsky and H. Gershinowitz, J. Chem. Phys. 1, 432 (1933). The corresponding estimates given by L. Pauling, J. Am. Chem. Soc. 54, 3570 (1930); L. Pauling and J. Sherman, J. Chem. Phys. 1, 606 (1933) are consistently approximately $0.25 \mathrm{ev}$ higher per bond due to the fact that these authors arbitrarily added one volt to the experimental heat of sublimation of carbon to allow for the difference between the ground ${ }^{3} P$ state and the excited i $S$ state (plus others) which take part in bond formation.

The assumption of equal bonding energies for all $\mathrm{C}-\mathrm{H}$ bonds in methane or methyl chloride may be misleading if applied to reactions such as

$$
\mathrm{CH}_{4}=\mathrm{CH}_{3}+\mathrm{H} \text { or } \mathrm{CH}_{3} \mathrm{Cl}=\mathrm{CH}_{3}+\mathrm{Cl} \text {. }
$$

The energy of reaction for these may be far different from the average bond energy. According to Mulliken, this assumption may hold fairly well for

$$
\begin{aligned}
& \mathrm{CH}_{\text {(rormal state) }}=\mathrm{CH}_{3} \text { ("valence state") }+\mathrm{H} \\
& \mathrm{CH}_{3 \text { ("valence state") }}=\mathrm{CH}_{2} \text { ("valonce state") }+\mathrm{H} \text {, etc. } \cdots
\end{aligned}
$$

where "valence state" (i.e., tetravalent) is perhaps 7 or 8
The experimental value $26.5 \mathrm{v}$ indicates that not only was the hope far from being realized but that the reaction yielding $\mathrm{Cl}^{+}$does not proceed unless enough energy is supplied to be sufficient to produce other fragments.

In the paper referred to above, Mulliken estimated the energy of removal of one of the bonding $\left[\sigma a_{1}\right]$ electrons in methyl iodide to be somewhat less than $14.4 \mathrm{ev}$. The corresponding value for methyl chloride would not differ much; it would presumably be little above that for the iodide. It is clear that such a removal may lead to the dissociation

$$
\begin{aligned}
\mathrm{CH}_{3} \mathrm{Cl}= & \mathrm{CH}_{3}{ }^{+}+\mathrm{Cl}+e^{-} \\
& (+ \text {excitational or kinetic energy }) .
\end{aligned}
$$

We shall therefore assume that the experimental appearance potential of $14.7 \mathrm{ev}$ for the $\mathrm{CH}_{3}{ }^{+}$ion is the energy necessary for the above reaction.

The ionization process in methane ${ }^{14}$ resulting in the formation of $\mathrm{CH}_{3}{ }^{+}$has been interpreted by Mulliken. ${ }^{15}$ The revised appearance potential value of Hipple and Bleakney ${ }^{8}$ indicates that the reaction

$$
\mathrm{CH}_{4}=\mathrm{CH}_{3}{ }^{+}+\mathrm{H}+e^{--}
$$

may be brought about by electron bombardment without imparting to the fragments more than about $0.5 \mathrm{ev}$ excitational or kinetic energy. This appears reasonable in view of the estimated ionization potential of $\mathrm{CH}_{3}{ }^{16}$ and the value chosen for the energy of breaking the initial $\mathrm{C}-\mathrm{H}$ bond. On the other hand, our results indicate (if the value chosen for the $\mathrm{C}-\mathrm{Cl}$ bond is correct)

volts above the normal state of $\mathrm{C}$ atom. Since it is probable that the normal state of $\mathrm{CH}_{3}$ is nearly the same as the valence state necessary for the reaction $\mathrm{CH}_{3}+\mathrm{H}=\mathrm{CH}_{4}$, the process (a) which would require about 6 volts, may be nearly identical with

$$
\mathrm{CH}_{4 \text { (normal state) }}=\mathrm{CH}_{3 \text { (normal stute) }}+\mathrm{H} \text {. }
$$

We shall therefore take for the energy of breaking the initial $\mathrm{C}-\mathrm{H}$ bond at 6 volts and for the $\mathrm{C}-\mathrm{Cl}$ bond, one electron volt less.

${ }^{14}$ Hogness and Kvalnes, reference 3.

15 R. S. Mulliken, J. Chem. Phys. 1, 492 (1933).

168.0 to $8.5 \mathrm{ev}$ as given R. S. Mulliken, reference 15 . also, J. Chem. Phys. 3, 521 (1935). This estimate is based on the following consideration. One can write for the configurations of

$$
\begin{array}{ll}
\mathrm{NH}_{3}: & 1 s^{2}\left[s a_{1}\right]^{2}[\pi e]^{4}\left[\sigma a_{1}\right]^{2},{ }^{1} A_{1} \text { I.P. of }\left[\sigma a_{1}\right]=11.1 \mathrm{ev} \\
\mathrm{CH}_{3}: & 1 s^{2}\left[s a_{1}\right]^{2}[\pi e]^{4}\left[\sigma a_{1}\right],{ }^{2} A_{1} .
\end{array}
$$

In the latter case, the ionization potential for the same type of electron should be 2.5 to 3 ev less than the former due to the smaller nuclear charge. 
that the excitational energy in the corresponding reaction (c) amounts to as much as $1.5 \mathrm{ev}$.

Further comparison with thermal data may be made. A simple calculation ${ }^{17}$ will show that the energy for the process

$$
\mathrm{CH}_{4}+\mathrm{Cl}=\mathrm{CH}_{3} \mathrm{Cl}+\mathrm{H} \text { is } 0.97 \mathrm{ev} \text {. }
$$

From the mass spectrometer experiments on methane and methyl chloride one can write:

For Eq. (d)

$$
\Delta E=\left\{\begin{array}{l}
15.5 \mathrm{ev} \text { (Hogness and Kvalnes) } \\
14.7 \mathrm{ev} \text { (Hipple and Bleakney) }
\end{array}\right.
$$

For Eq. (c)

$$
\Delta E=14.7 \mathrm{ev} \text { (Bauer and Hogness) }
$$

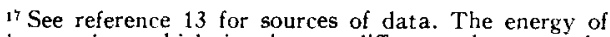
this reaction, which involves a difference between the energies of formation of methane and methyl chloride is independent of the value chosen for the heat of sublimation of carbon or of any assumptions regarding the relative values of bonding energies, and hence is much more reliable than any of the calculations referred to in 13 . resulting in

$$
\mathrm{CH}_{4}+\mathrm{Cl}=\mathrm{CH}_{3} \mathrm{Cl}+\mathrm{H} \quad 0.8 \text { or } 0.0 \mathrm{ev}
$$

depending on the value chosen for the energy of reaction (d).

The obvious conclusions are: (1) If the reactions (c) and (d) are analogous (it is not essential that they be as simple as written; it is only necessary that the same amount of excitational energy occur in both), the Eq. (e) may rightfully be deduced. Then, it is clear, either the second value of (d) or the one given for (c) is incorrect. Or, as was suggested in the preceding paragraph, (2) the first value of (d) may be too high and the reactions are not entirely analogous. There is then no justification for (e) since the products in (c) are excited to an extent of approximately one volt more than in (d).

The authors wish to thank Professor Mulliken for a number of helpful discussions pertaining to questions presented in section IV.

\section{Monomolecular Films of Molecules Which Lie Flat on the Surface of Water}

\section{Surface Pressures and Potentials of Films of Long Molecules : Polymers of $\omega$-Hydroxy Decanoic Acid}

William D. Harkins, Everett F. Carman and Herman E. Ries, Jr., University of Chicago

(Received September 3, 1935)

Film pressures and surface potentials have been measured for a series of linear polymers of $\omega$-hydroxy decanoic acid, ranging in molecular weight from 780 to 25,200 . The molecules are oriented parallel to the surface. An improved form of the apparatus of Harkins and Fischer was used. The chief modification in the procedure was the use of two radioactive polonium electrodes over the film. The pressure-area relations show that the area per molecule in the condensed films is nearly proportional to the molecular weight and that the compressibility of the films is large. The film density is found to be lowest for the polymers of smallest molecular weight. The films are not very sensitive to changes in hydrogen ion concentration, but on changing from an acid to an alkaline substrate the films in the expanded state give higher molecular areas at given pressures, and the pressures for film collapse are considerably higher. Dimensions of the molecules are calculated and a complete table of mean values is included. The vertical cross section of the molecules varies between 19.4 and 19.9 sq. A or is nearly the same as the horizontal cross section (20.5) of vertically oriented molecules such as stearic acid. Some differences between films with perpendicular orientation to the surface and those with parallel orientation are tabulated. The surface potentials of the polymer films rise to between 400 and $450 \mathrm{mv}$, although the surface density of the polar groups is only $1 / 3$ to $2 / 5$ that of stearic acid type films, which also give potentials of about $400 \mathrm{mv}$. The contribution of each polar group is, therefore, considerably greater than that of the dipole of the vertically oriented stearic acid molecule. The surface potential of a condensed film was found to increase about 13 percent less rapidly than the surface density of the dipoles. 